Revista Iberoamericana de Ciencias de la Actividad Fisica y el Deporte

\title{
DESARROLLO COMUNICACIONAL EN LOS CLUBES DE FÚTBOL ESPAÑOLES. ESTUDIO DE CASO: SEVILLA FC
}

PROFESSIONALIZATION AND COMMUNICATION DEVELOPMENT IN SPANISH FOOTBALL CLUBS. CASE STUDY: SEVILLA FC

Lobillo, G.

${ }^{1}$ Gema Lobillo. Profesora asociada del Dpto. Comunicación Audiovisual y Publicidad de la Facultad de Ciencias de la Comunicación de la Universidad de Málaga (España). gmlobillo@uma.es

Código UNESCO: 590214 Política de la Información; 591003 Prensa; 591002 Medios de Comunicación de Masas; 591001 Información

Clasificación Consejo de Europa: 16. Sociología del deporte

Recibido el 22 de diciembre de 2017

Aceptado el 27 de febrero de 2018

Correspondencia:

Gema Lobillo

gmlobillo@uma.es

\section{RESUMEN}

La gestión de los departamentos de comunicación en los clubes de fútbol se ha convertido en algo imprescindible para el funcionamiento de estas entidades, ya que estos han experimentado un gran crecimiento y profesionalización en la última década, integrándose como parte fundamental del organigrama e impulsado por las necesidades y beneficios de una correcta transmisión de la identidad corporativa y gestión empresarial, además de complementar otras áreas.

El objetivo de este estudio es realizar una panorámica de la situación actual del área de comunicación en estas entidades deportivas, así como realizar una comparativa entre clubes en el tiempo. Concretamente, el club donde basaremos el análisis será el Sevilla Fútbol Club (en adelante Sevilla FC). Para ello, se han utilizado una metodología centrada en el estudio del caso y apoyada en datos de otras investigaciones realizadas a través de un cuestionario a los clubes de fútbol y las entrevistas en profundidad.

Palabras clave: Departamento de Comunicación, dirCom; fútbol, comunicación, medios de comunicación. 


\section{ABSTRACT}

The management of communication departments in Football clubs has experienced exponential growth and a rise in professionalism in the last decade. It has become a fundamental part of the entity's identity and organizational structure, driven by the needs and benefits of a correct transmission of corporate identity and business management.

The aim of this study is to provide an overview of the current situation of the communication area in these sports organizations, as well as to make a comparison between clubs in time. Specifically, the club where we will base the analysis will be the Sevilla Fútbol Club (hereinafter Sevilla FC). To do so, a methodology based on the Case Study has been used and supported by data from other research conducted focusing on the Questionnaire to football clubs and in-depth interviews.

Key words: Communication Departaments, dirCom communications, football, communication, mass media.

\section{INTRODUCCIÓN}

Son diversos los factores que han forzado la profesionalización de los departamentos de comunicación, como las tecnologías, la mercadotecnia o el poder de transcender cualquier tipo de frontera. La comunicación es para Moragas ${ }^{1}$, como uno de los cuatro actores del deporte moderno junto a los clubes y deportistas, patrocinadores y administración.

En este contexto las entidades deportivas se plantean la obligada necesidad de implantar la comunicación como valor añadido a su gestión y la necesidad de abarcar este ámbito para acompañar su estrategia empresarial puesto que no podemos olvidar que están constituidas como sociedades anónimas deportivas.

El Sevilla FC es el club de la Liga de Fútbol Profesional (en adelante LFP) que nos sirve como muestra para realizar el estudio comparativo mediante el cual podremos comprobar la estructura y funciones del gabinete de comunicación y sus figuras fundamentales. Teniendo en cuenta que las funciones que integran este departamento siempre viene condicionado por la estructura del club, sus particularidades y necesidades obtendremos la dinámica y metodología de uno de los grandes clubes de fútbol a nivel mundial.

La gestión comunicativa se presenta fundamentalmente dentro de las estrategias de las organizaciones, especialmente en la relación con sus públicos objetivos, por lo que resulta imprescindible plasmar y analizar el papel de los departamentos desde el seno de la entidad y como se desarrollan dentro de las funciones directivas.

Para Capriotti ${ }^{2}$ «los departamentos de comunicación desempeñan un papel fundamental para entender la apuesta que hacen para gestionar un valor 
intangible como es la comunicación». A lo que añade Olabe-Sánchez ${ }^{3}$ que «en la actualidad estos departamentos se refuerzan con otras disciplinas como el marketing, o se integran en las direcciones de comunicación».

Concretamente en el fútbol, ya que se trata de la principal fuente de atención para los periodistas deportivos y generalizados, la comunicación adquiere un rol de suma importancia. Desde la comunicación diaria (resultados, lesiones, novedades, etc.) hasta que más específica. Por ello, se hace necesaria una relación club-periodista, donde el servicio periodístico requerido debe de ser continuo.

En muchas organizaciones como afirman Carrillo y Tato $^{4}$ se da la circunstancia de que el departamento de comunicación se ocupa de distintas actividades que le corresponden a otras áreas, por lo que resulta confuso definir sus funciones y el término queda sujeto a diferentes interpretaciones.

El caso de estudio del club de fútbol para esta investigación se centrará en el Sevilla FC, por lo que hay que conocer una breve historia de la entidad deportiva para poder desarrollar el estudio de forma adecuada. Podríamos hacer una línea temporal que describe las distintas etapas del club de fútbol:

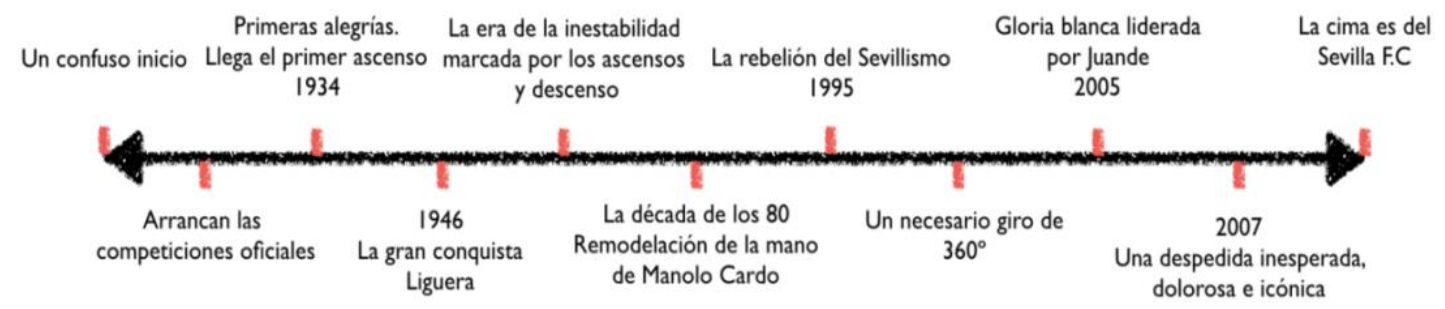

Imagen 1.- Línea temporal histórica del Sevilla FC. Fuente: Elaboración propia

Es importante señalar la llegada de las tecnologías de la información, ya que han significado un progresivo incremento de los medios de comunicación propios en la última década. Medios que en algunos casos han permitido que la entidad llegue a su público objetivo con una mayor efectividad y con costes insignificantes. Favorece a que la globalización siga su cauce y se logre la creación de una marca por parte del club, además de reforzar el sentimiento de pertenencia de los aficionados.

Agudo y Toyos $^{5}$ en 2003 empezaron a advertir que Internet era la vía que concreta mayores esperanzas de cara a una remodelación de la comunicación de las organizaciones.

La evolución tecnológica ha traído consigo nuevas formas de ingresos eficaces para los clubes, además de herramientas y canales para mantener un 
contacto continuo y recíproco con todos sus seguidores. Olabe-Sánchez $z^{6}$ estudia los fundamentos de la nueva comunicación, donde la clave está en analizar a los públicos, saber que precisan y adaptarse a sus necesidades y sugerencias. A esto añade, «la importancia que cobran las nuevas tecnologías reside en saber que precisa nuestro público para conseguir los objetivos, ya que permiten escuchar e interactuar con ellos».

«Entendemos el fútbol como un negocio, comprenderemos los profundos cambios que ha sufrido convirtiendo el deporte en una industria. Históricamente la televisión desde finales del S.XX ha tenido el papel fundamental emitiendo el espectáculo de masas y como hemos nombrado anteriormente con la llegada del S.XXI todo está conectado y vinculado con las Tecnologías de la Comunicación, adquiriendo otra dimensión y entrando en una esfera pública y accesible imperada por el rendimiento económico", según Ginesta? ${ }^{7}$.

Enfocándonos más en las áreas estructurales del club, las TIC han modificado el comportamiento de los departamentos de comunicación haciendo inevitable realizar una sinergia entre las acciones offline y online.

Para Túñez ${ }^{8}$ «las relaciones con los medios de comunicación y con nuestras audiencias propias se ven modificadas donde ya no solo se interactúa sino se comportan todos los puntos del segmento como generadores de contenidos».

Por lo tanto vamos a destacar los diferentes esfuerzos que realiza el Sevilla FC por informar, vender, publicitar y entretener en el mundo 2.0. Este cambio en el paradigma comunicativo fuerza la adaptación e incorporación de nuevas herramientas de comunicación, a través del departamento de comunicación.

Los gabinetes de comunicación son considerados como fuentes activas que cubren todos los aspectos comunicativos de las organizaciones (internos/externos) y transmiten de ellas una imagen positiva a la sociedad al tiempo que tratan de influir en la opinión pública9 .

Haciendo referencia a las TIC, Olabe-Sánchez ${ }^{10}$ expone que «la comunicación interactiva en los clubes de fútbol responde a las características diferentes a la empleada en otros sectores empresariales. Lo destinatarios de esta comunicación son, fundamentalmente, seguidores con fuerte arraigo, compromiso e identificación con estas entidades». Precisamente el deporte se está convirtiendo en ese mundo 2.0 de la sociedad de la información revolucionado, hiperconectado, global, inmediato y complejo, y ha transformado las relaciones sociales a través de la combinación de ceros y unos. Cualquier organización debe tener en cuenta que cualquier acto que realicen significa y, por tanto comunica, transmite identidad e imagen ${ }^{11}$. Un factor que se incrementan por la instantaneidad y alcance de la era digital.

No obstante, como bien considera Álvarez y Caballero ${ }^{12}$, «todas las organizaciones, sea cuál sea su carácter, son conscientes de que sólo el éxito sino incluso la supervivencia está condicionada por el desarrollo de una acción 
planificada, porque la entidad no es para la sociedad sino la imagen que de ella ofrecen, principalmente, los medios de comunicación».

\section{MÉTODO}

Para esta investigación ha empleado una combinación de métodos de distinta índole (cualitativa/cuantitativa). Por un lado, se emplearon herramientas de carácter cualitativo, que tienen como finalidad, "poner énfasis en estudiar los fenómenos sociales en el propio entorno que ocurren, dando primacía a los aspectos subjetivos de la conducta humana sobre las características objetivas, explorando sobre todo, el significado del acto humano ${ }^{13}$. El estudio de campo mediante la revisión bibliográfica nos proporcionara un contexto cualitativo sobre el que apoyar la investigación. Además, analizamos el trabajo de investigación realizado por Lobillo ${ }^{14}$, titulado "Análisis de la estructura departamental de comunicación en los clubes de fútbol españoles. Temporada 2010-11».

Los resultados del estudio de Lobillo han sido comparados y contrastados con los resultados obtenidos de la entrevista en profundidad realizada a Jesús Gómez, anteriormente mencionada. Esta técnica se eligió con la finalidad de reunir información muy minuciosa de una muestra pequeña pero a su vez muy representativa. La entrevista se planteó con unos puntos fijos a tratar, resultados estudio Lobillo, y otra parte no estructurada donde el entrevistado pudo tratar temas de interés de carácter abierto.

Con la combinación de estos métodos, hemos fundamentado, contrastado y corroborado los resultados obtenidos. Mediante la revisión bibliográfica de diferentes autores y trabajos sobre la comunicación deportiva realizaremos un trabajo de análisis e interpretación de datos, siguiendo especificaciones expresadas orientadas a resolver las cuestiones planteadas previamente.

\section{RESULTADOS}

Este trabajo parte con la finalidad de conocer el funcionamiento y la estructura de los departamentos de comunicación en el mundo del deporte y concretamente en el fútbol en la actualidad, así como han evolucionado. Para ello se ha escogido como muestra el Sevilla FC.

El primer punto a tratar en la entrevista a Gómez consistió en la importancia que poseen los departamentos de comunicación en el mundo del fútbol y dentro de las entidades deportivas, a lo que el profesional lo definió como «una labor fundamental hoy día, porque esta comunicación va más allá de las relaciones comunes con los medios. los departamentos de comunicación cumplen primero una función importante para que la información llegue directa al aficionado sin pasar por el resto de los medios». Por otro lado es cierto que el Sevilla FC como medio de comunicación propio lógicamente no va a comunicar nada negativo de la entidad.

Lobillo ${ }^{14}$ obtuvo como resultado de los clubes estudiados que «el $54 \%$ de los mismos lo denominan gabinete o departamento de comunicación»

38 DESARROLLO COMUNICACIONAL EN LOS CLUBES DE FÚTBOL ESPAÑOLES. ESTUDIO DE CASO: SEVILLA FC 
representando un alto porcentaje, frente un «segundo lugar, con un $23 \%$ de los clubes, el término "Comunicación" lo unen con otra área o ámbito, tales como relaciones externas, imagen o proyectos internacionales", un 15\% lo denominan como departamento de prensa relacionándolo con las funciones junto a los medios de comunicación y por último el $8 \%$ realizan una denominación conjunta de comunicación y prensa.

En el seno del Sevilla FC se le denomina «departamento de comunicación», reconociendo que en el pasado se le llamaba «departamento de prensa» pero el término ha evolucionado creando esta diferenciación y siendo el área de prensa controlada por el departamento de comunicación y gestionada por el dirCom.

Lobillo $^{14}$ buscó la ubicación exacta de este departamento en el organigrama de la empresa ya que facilitaría información sobre el grado de interactuación con la alta dirección. Como resultado obtenemos que los clubes se relacionan con casi todos los departamentos del club. Las áreas comunicativas dependen de la dirección general en un $46 \%$. Dependen en un $31 \%$ de la presidencia y en un mismo porcentaje $31 \%$ de los consejeros delegados. Por último una muestra desprende que dependen el $15 \%$ de otro tipo de direcciones, tales como la dirección de desarrollo y dirección general de organización y gestión. A lo que el dirCom del Sevilla FC añade que el departamento de comunicación en el club se encuentra al mismo nivel que otros departamentos como puede ser, marketing, instalaciones, seguridad, administración, ... buscando siempre una homogenización.

Es de gran importancia analizar la relación que posee el dirCom con otras áreas de la entidad y el grado de participación con los que se relaciona, para ello Lobillo determino tres categorías: alta, media y baja participación.

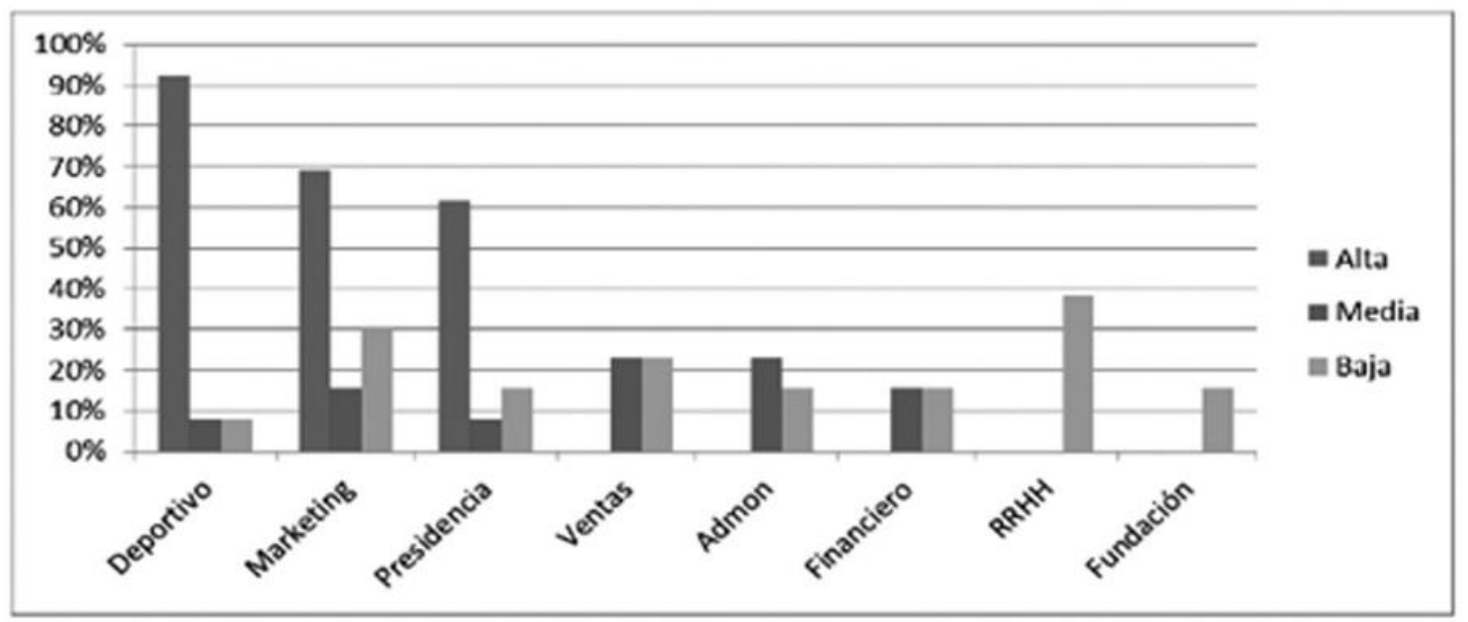

Imagen 3.- Relación de las áreas con el departamento de comunicación de los clubes de fútbol. Fuente: Lobillo 
Esta misma forma de cuantificar los resultados en baja, media y alta dependiendo de la relación que se posee con cada departamento se aplicó para plasmar las actuales relaciones del departamento de comunicación del Sevilla $\mathrm{CF}$, cuyos resultados:

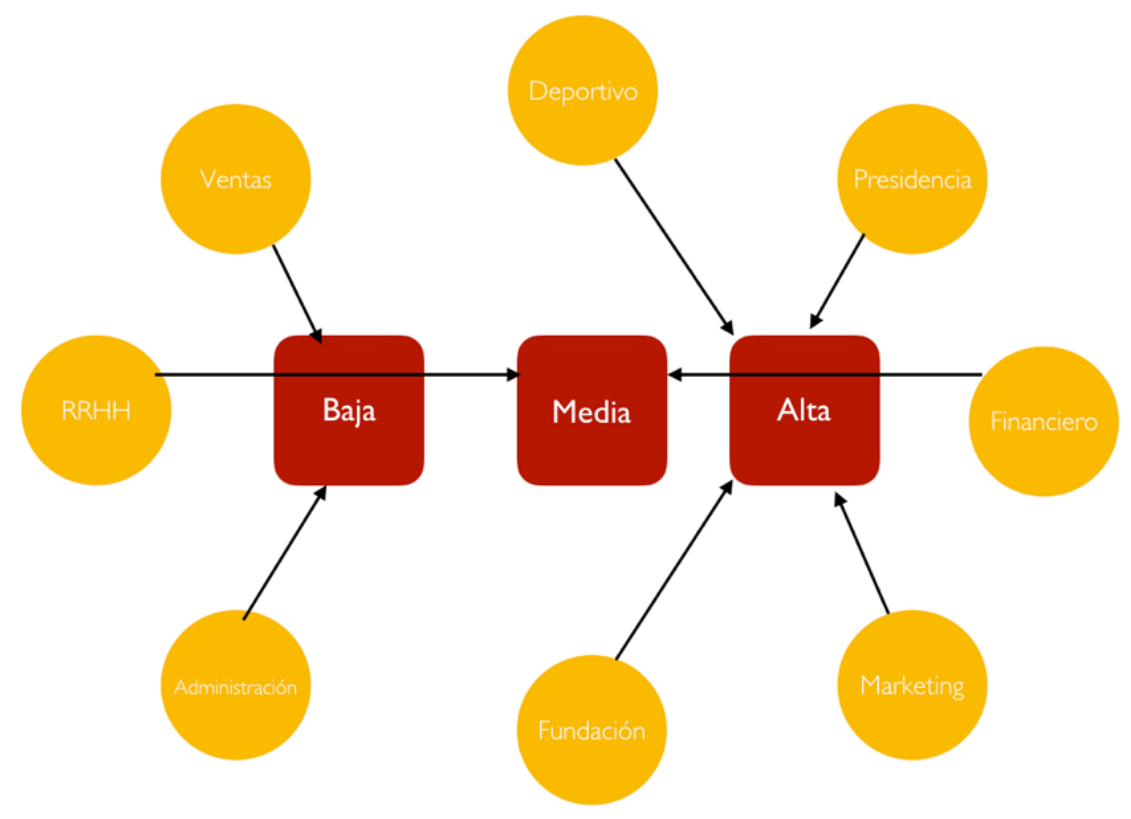

Imagen 4.- Relación del departamento de comunicación del Sevilla FC con el resto de las áreas del club. Fuente: Elaboración propia

Comparando los resultados de ambos estudios, vemos como se asemejan en un porcentaje medio, siendo el club analizado un elemento diferenciador frente a la muestra elegida en el otro estudio. Coinciden en una relación alta entre los departamentos deportivos, marketing y presidencia, a excepción de la fundación que la que el Sevilla FC posee un relación alta ya que son los responsables de canalizar toda la información de la fundación. En la participación media, encontramos en la investigación de Lobillo, el departamento de administración (23\%) y ventas (23\%), siendo ambos departamentos clasificados con una participación baja por el departamento de comunicación del Sevilla FC. Con una pertinencia media, el club marca recursos humanos y financiero siendo ambos catalogados con una colaboración baja por la muestra de clubes analizados en el estudio de Lobillo.

Lobillo $^{14}$ analiza las principales funciones de los responsables de comunicación realizan en el seno de los clubes de fútbol, afirmando las siguientes: un 77\% confirma que se realiza la función de liderazgo y motivación en la participación de su equipo de trabajo, además de fijar medidas de control y seguimiento de las acciones. Mediante la entrevista pudimos corroborar esta 
información, mostrándose el club partidario de una comunicación bidireccional y transversal que empape a toda la entidad.

Gómez, como fuente oral de nuestro estudio, añade que desde el departamento se establecen una serie de herramientas fijas para el seguimiento y control de tareas internas como externas, bajo una supervisión de todo el equipo. Por otro lado con respecto a las tareas cotidianas afirma que no son revisadas, ya que se posee confianza en los responsables de área dentro del departamento siendo en concreto cuatro; los cuales poseen autonomía plena.

El $69 \%$ de los clubes analizados confirman que la función de validación de resultados y gastos del departamento es responsabilidad de la propia área incluyendo la supervisión de acciones y definición de funciones de cada miembro. El Sevilla FC confirma los datos, sumándose a esta muestra ya que la coordinación de tareas y supervisión tanto de presupuesto como de acciones recae sobre el responsable de departamento y el personal al que se le delega dicha función.

El $46 \%$ de la muestra afirma que desempeñan una función de reporte de los resultados a la directiva y presidencia. En el departamento analizado se realizan reuniones cada quince días y si es necesario de manera semanal para informar sobre movimientos, novedades y resultados obtenidos, siendo el dirCom el máximo responsable de esta función.

Con un porcentaje del $31 \%$, plasma la función de aprobación y contratación de consultoras o investigadoras externas forma parte de esta área. El dirCom del Sevilla FC afirma que no hacen uso de consultoras externas, uniéndose a la muestra mayor del estudio de Lobillo. Afirma que desde el departamento se encargan prácticamente de todo, sin delegar funciones a empresas externas salvo casos muy puntuales.

Lobillo fija las relaciones del club con los profesionales de los medios de comunicación como tarea mas importante. Por medio de la entrevista obtuvimos la forma en la que el Sevilla FC se organiza con los principales medios de comunicación y líderes de opinión.

Con el paso del tiempo las tecnologías han ido empapando todas las secciones empresariales, muestra de ello es que el dirCom mantenía como herramienta fija de comunicación los correos electrónicos y actualmente se utiliza directamente la aplicación WhatsApp con diferentes grupos, con los principales responsables de los medios, por ser una herramienta mas directa y rápida. También se utilizan los grupos de WhatsApp como herramienta de comunicación interna (con el propio personal del departamento y otras áreas como es televisión e imagen, radio y prensa escrita y redactores webs).

En cuanto al seguimiento de noticias diarias, el club tiene esta tarea asignada a una persona. Además se revisan continuamente las páginas web mediante un filtrado y las redes sociales, en busca de la obtención de la tonalidad de las publicaciones. 
Antes las relaciones con los medios y el público externo eran más menos complicadas, en palabras del dirCom: «Hoy día, las redes sociales lo manipulan todo, por eso tenemos que hacer también una labor de control y de desmentir informaciones que muchas veces con frialdad no tienen la vista puesta en la realidad».

Como balance de la entrevista se concluyó que la inmersión en el mundo de las TIC, Gómez añade; «hace diez años no sería viable que nosotros pudiéramos hacer para una televisión local un programa sobre cuando nuestro equipo juega fuera, y hoy día y gracias a Internet y las innovaciones aplicadas podemos llegar a nuestros aficionados hasta y desde cualquier punto».

Las relaciones públicas ocupan el $9 \%$ del total y la comunicación en situación de crisis un 7\%. En estos aspectos Gómez quiso darle importancia a estas funciones, ya que «son factores que en muchos casos pasan desapercibidos en el mundo empresarial y organizativo cuando son áreas que marcan la diferencia y poseen una vital importancia».

El porcentaje restante lo ocupan con un $5 \%$ las relaciones con peñas y aficionados, otro $5 \%$ en el ámbito de la responsabilidad social, el $4 \%$ dedicado a auditorias de imagen y reputación corporativa y por último la comunicación financiera representa el 3\%. Estas últimas labores citadas también forman parte del planning del Sevilla FC, siendo conscientes de la necesidad de su desarrollo. Resaltando la necesidad de las auditorias, donde Gómez añade que «es importante conocer de dónde venimos y hacia dónde vamos, sino tenemos claro esto nunca se puede avanzar. Por lo tanto el control y análisis de la efectividad de nuestras acciones y el efecto que provoca en el público objetivo es fundamental».

De la muestra de clubes españoles elegida en el estudio se concluyó que los departamentos de comunicación están compuestos de una media de cinco personas, realizando cada de ellas tareas y funciones diferenciadas, pero con la particularidad de que todas deben tener capacidades suficientes para desarrollar todas las competencias del área comunicativa. Lobillo ${ }^{14}$ accede a estas conclusiones conociendo los tipos de acciones de comunicación que desarrollan, observando que por término medio, una sola persona acomete cada tipo de comunicación o varios tipos de comunicación a la vez. Lo muestra mediante algunas matizaciones: «que las relaciones con los medios de comunicación tienen una media más alta, contando con casi dos personas $(1,79)$ de media, junto con la comunicación de imagen corporativa $(1,50)$ y la comunicación comercial, marketing y publicidad $(1,33)$ o la comunicación de las TIC, relaciones públicas o comunicación interna, éstas últimas con 1,25 personas de media. El resto no llegan a una persona exclusivamente para cada tipo de comunicación ». Actualmente el departamento de comunicación del Sevilla FC está formado por 33 trabajadores que poseen un contrato en vigor, sin contar a los colaboradores.

Según las informaciones recabadas por el estudio de Lobillo, apreciamos que los departamentos de comunicación están compuestos por licenciados en ciencias de la información (79\%), siendo de estos un $47 \%$ licenciados en 
periodismo, 22\% licenciados en publicidad y RRPP, 10\% comunicación audiovisual, acompañados por licenciados en marketing (8\%), licenciados en económicas y empresariales (4\%), licenciados en derecho $(2 \%)$ y el resto $(8 \%)$ está formado por otras titulaciones.

Con vistas al futuro y a la base en la que se fijan para la actual contratación en los departamentos, hemos podido comprobar mediante los testimonios de trabajadores del departamento del Sevilla FC, que las materias que mejor se están posicionando son las relacionadas con el mundo digital. El conocimiento del marketing digital, redes sociales, programación, idiomas, sin olvidar los fundamentos básicos de las materias en comunicación e información.

El primer parámetro estudiado en el artículo de Lobillo son las redes sociales, ofreciendo que el $77 \%$ de los clubes estudiados están presentes en distintas Redes Sociales (Facebook, Twitter, YouTube, etc.). Contando con el año de publicación del estudio (2012) y el avance de las tecnologías es evidente que todos los clubes de la muestra estudiada por la autora a día de hoy poseen perfil al menos en las principales redes sociales.

Paralelas a este mismo avance encontramos la aparición de televisiones oficiales a causa de la facilidad de difusión y la escasa inversión económica que requiere. Lobillo demostró que desde los propios departamentos de comunicación se fomentan y editan las noticias de actualidad canalizándolas mediante estos soportes. El $46 \%$ de las entidades deportivas estudias posee un canal propio de televisión cuyo público principal es el aficionado. El 31\% posee emisora de radio oficial. El $23 \%$ publican un periódico oficial, dirigido al aficionado pero con un coste por parte del consumidor, aunque actualmente se editan de manera digital por lo que se ha conseguido eliminar el costo del papel. El Sevilla FC es un club muy concienciado con que el principal activo, fuera de lo meramente deportivo, es el aficionado. Por eso, desde el departamento de comunicación se le ofrece a los medios propios una posición privilegiada.

Por último y para completar este abanico de soportes y medios propios tenemos la inmersión en el mundo de las redes sociales, que están experimentando modificaciones en el modelo, aportando un continuo flujo de información y de noticias que el departamento de comunicación publica en la web oficial, de esta forma se produce una generación de contenidos propia. El siguiente paso fue el comienzo de la traducción de las redes sociales a más de diez idiomas, contribuyendo al crecimiento de seguidores y a la comunicación de contenidos de calidad.

Uno de los aspectos planteados al dirCom del Sevilla FC fue la profesionalización que se estaba alcanzando por parte de los departamentos de comunicación en los clubes de futbol. Según el dirCom del Sevilla FC, "esta área se debe a que cada día se parece más a una organización comercial que vende una marca». 


\section{DISCUSIÓN}

Podemos concluir que el departamento de comunicación a día de hoy posee una posición equivalente a la del resto de áreas de la entidad, pero privilegiada por el contacto directo y continuo con la cúpula del organigrama. Gracias a esta estructura jerárquica el departamento goza de relaciones productivas y bidireccionales con los demás departamentos, en especial con el deportivo, presidencia, marketing y fundación que nutren a la organización tanto de manera interna como externa.

En este mismo nivel el dirCom posee diferentes funciones, como validación de resultados y funciones presupuestarias, revisión de la información, liderazgo y motivación, reporte de resultados, aprobación de propuestas, contratación de consultoras externas, mantenimiento de la imagen e identidad corporativa y apoyo/participación con el resto de áreas.

Observando la escasa madurez de las tecnologías y el camino que viene marcando hasta su implantación en los departamentos de comunicación en los clubes de fútbol, podemos observar que su inclusión e instauración ha sido lenta, utilizándose principalmente para el contacto con los medios de comunicación y las comunicaciones externas. En definitiva podemos coincidir que su inclusión ha sido muy favorecedora gracias a la instantaneidad que posee, el poder de abaratar costes, eliminación de barreras con los públicos y la alta difusión y alcance que lleva aparejada como característica principal.

Respecto a la antigüedad del departamento podemos añadir que poseen una edad media de diecinueve años y con una media de veinticinco trabajadores en los clubes profesionales de élite. En cuanto a la formación del personal de departamento encontramos un alto grado de formación, observando los resultados de Lobillo y añadiendo la muestra estudiada, un $85 \%$ del personal serían graduados frente al $15 \%$ diplomados. En lo que a especialización en materia se refiere, encontramos que las carreras de periodismo, publicidad y relaciones públicas, comunicación audiovisual y marketing son las que más apreciamos como especialización en la composición del departamento.

Los medios propios del club sufrieron un incremento con la aplicación de las TIC, dejando como resultado instrumentos mediáticos de gran alcance que en todo caso ayudan a ampliar y llegar a nuestro público objetivo en su totalidad y reforzar el sentimiento de pertenencia. Las herramientas y plataformas más frecuentadas por los clubes son las redes sociales, siendo Facebook, Twitter, Instagram y YouTube las más utilizadas por este tipo de perfiles. Las páginas web son el pilar de la información del club, siendo esta la que nutre el resto de herramientas de comunicación. En este contexto actual se siguen manteniendo y con un mayor presupuesto y poder, las televisiones y radios ambas corporativas con contenidos propios al 100\%. En el caso de los periódicos oficiales los encontramos en su totalidad en formato digital, de este mismo modo se difunden las revistas y publicaciones oficiales publicadas por el club.

En el caso del Sevilla FC, por medio de su departamento de comunicación y la gestión del mismo podemos ver la correlación que tiene éxito/crecimiento 
haciéndose evidente que el interior repercute en el exterior. Muestra de que cuando se trabaja bien en el seno de la entidad, es cuestión de tiempo que lleguen los resultados. Fijándonos en el personal, la forma de organizarse y trabajar, especialización de los trabajadores, el Sevilla FC se trata de un club que innova en el campo de la comunicación y lucha por acercarse al aficionado, entendiendo a éste como uno de los principales valores de la entidad. Por lo que por sus contenidos y presencia podríamos decir que se trata de un club vanguardista en tema de comunicación.

\section{REFERENCIAS}

1.- De Moragas, Miquel. Comunicación y Deporte en la era Digital, Barcelona. Olimpismo y deporte: CEO-UAB; 2007.

2.- Capriotti, P. La imagen de la empresa. Estrategia para una comunicación integrada. Barcelona: El Ateneo; 1992.

3.- Olabe-Sánchez, F. La comunicación no convencional en los clubes de fútbol. Pensar la Pub. Rev. Intern. de Invest. Public. 2009; Vol3, N1. Disponible en http://revistas.ucm.es/index.php/PEPU/article/view/PEPU0909120121A/15348

4.- Carrillo, Ma . Y Tato, J. La nueva dimensión de la comunicación empresarial en el entorno de los activos intangibles. La comunicación espiral. Raz y Pal. 2004. Disponible en: http://www.razonypalabra.org.mx/anteriores/n39/carrillotato-html

5.- Agudo, A. y Toyos, F. Marketing de fútbol. Madrid: Pirámide; 2003.

6.-Olabe-Sánchez, F. La comunicación no convencional en los clubes de fútbol. Pensar la Pub. Rev. Intern. de Invest. Public. 2009; Vol3, N1. Disponible en http://revistas.ucm.es/index.php/PEPU/article/view/PEPU0909120121A/15348

7.- Ginesta, X. El fútbol y el negocio del entretenimiento global. Los clubes como multinacional del ocio. Com. y Soc.2011 vol. XXIV, n. 1, pp.141-166.

8.- Túñez. M. Los periódicos en las redes sociales: audiencias, contenido, interactividad y estrategias comerciales. Estud. Leng. Period.; Vol.18, N1. Disponible en https://revistas.ucm.es/index.php/ESMP/article/viewFile/39367/37913.

9.- Ramírez, T. Gabinetes de comunicación. Funciones, disfunciones e incidencia. Barcelona: Bosch Comunicación; 2002.

10.- Olabe-Sánchez, F. La comunicación no convencional en los clubes de fútbol. Pensar la Pub. Rev. Intern. de Invest. Public. 2009; Vol3, N1. Disponible en http://revistas.ucm.es/index.php/PEPU/article/view/PEPU0909120121A/15348

11.- Costa, J. La comunicación en acción - Informe sobre la nueva cultura de la gestión. Barcelona: Paidós; 1999.

12.- Álvarez, T. y Caballero, M. Vendedores de imagen. Los retos de los nuevos gabinetes de comunicación. Barcelona: Paidós; 1997. 
13.-Ruiz Olabuénaga, J. Aristegui, I.Melgosa, L. Como elaborar un proyecto de investigación social. Bilbao: Universidad de Deusto; 1998.

14.- Lobillo, G. Análisis de la estructura departamental de comunicación en los clubes de fútbol españoles. Temporada 2010-11. En: Carretón, M.C. y Matilla, K. La conducta interactiva de los públicos para las relaciones eficaces. Las relaciones en la sociedad y en las redes sociales. Málaga: Unión de Editoriales Universitarias Españolas (UNE); 2012.

Referencias totales citadas: 14

Referencias citadas correspondientes a la Rev lb CC Act Fis Dep: 0 